\section{Desfechos relacionados à gravidez em áreas contaminadas, SP, Brasil}

\section{Pregnancy outcomes in contaminated areas, SP, Brazil}

Mariana Tavares Guimarães ${ }^{1,11}$

Michele Granato Cunha"

Daniele Pena Carvalho'

Tatyana Sampaio',"1

Alfésio L.F. Braga' ${ }^{\prime \prime \prime \prime}$

\section{Luiz Alberto Amador Pereira',"1}

'Faculdade de Medicina da Universidade de São Paulo - FM/USP.

"Programa de Pós-graduação em Saúde Coletiva da Universidade Católica de Santos - UNISANTOS.

Trabalho realizado pela Universidade Católica de Santos - UNISANTOS.

Conflito de interesse: nada a declarar.

Financiamento: Este trabalho fez parte de um projeto mais amplo intitulado "Estudo Epidemiológico na população residente na Baixada Santista - Estuário de Santos: Avaliação de indicadores de efeito e exposição a contaminantes ambientais", financiado pelo Conselho Nacional de Pesquisa - CNPq Edital 50/2005 - número 402663/2005-5.

Correspondência: Mariana Tavares Guimarães. Alameda Itu, 395 Apto. 81 - Jardim Paulista - São Paulo, SP CEP 01421-000. Email: marianatguimaraes@usp.br

\section{Resumo}

Objetivo: Estimar e comparar a prevalência dos eventos relacionados à gravidez (engravidar, baixo peso de nascimento, parto prematuro, aborto espontâneo, natimortalidade, malformações congênitas e gemelaridade) em populações exposta e não-exposta aos contaminantes ambientais na região do estuário de Santos e São Vicente. Métodos: $\mathrm{O}$ estudo fez parte de um amplo projeto financiado pelo CNPq, que teve como objetivo estimar os efeitos à saúde associados à exposição aos contaminantes ambientais entre os moradores da Baixada Santista. O estudo transversal avaliou dois bairros do município de São Vicente, próximos a uma área contaminada, e um bairro no município de Bertioga, área controle. Para a obtenção dos dados foi aplicado um questionário estruturado e pré-testado em 236 domicílios em São Vicente e 251 domicílios em Bertioga. Para avaliar associações entre a área e as variáveis qualitativas utilizouse o teste qui-quadrado ou teste exato de Fisher; para avaliar as diferenças entre as variáveis, o teste $t$ de Student ou o teste de comparação de duas proporções, e adotado nível de significância de 5\%. Resultados: Houve associação significativa entre morar em Bertioga ( $p=0,01)$ e o número de gestações ocorridas nos últimos cinco anos. Em São Vicente, 64 (28,3\%) mulheres em idade fértil engravidaram, enquanto em Bertioga foram 109 (38,8\%). Não houve associações estatísticas significativas nas áreas com os demais desfechos da gravidez avaliados. Conclusões: As prevalências e as razões de chances prevalentes de baixo peso ao nascer, nascimentos prematuros e abortos espontâneos foram maiores nas áreas contaminadas, sem associações significativas. A evidência do estudo de diminuição do número de gestações na área contaminada reforça a necessidade de aprofundamento de estudos na região do estuário de Santos e SãoVicente.

Palavras-chave: Gravidez. Poluição ambiental. Compostos organoclorados. Metais pesados. Baixo peso ao nascer. Prematuridade. 


\section{Abstract}

Objective: Estimate and compare prevalence of events related to pregnancy (pregnancy, low birth weight, premature delivery, spontaneous abortion, stillbirth, congenital malformation, and multiple births) in populations exposed and non-exposed to environmental contaminants in Santos and São Vicente Estuary. Methods: This study was part of a large project financed by $\mathrm{CNPq}$, which aimed to estimate health effects associated with environmental area, contaminants exposure among individuals of the Baixada Santista region. This cross-sectional study evaluated two neighborhoods of São Vicente near a contaminated area, and one neighborhood of Bertioga, the control area. A structured and previously tested questionnaire was applied at 236 households in São Vicente and 251 households in Bertioga in order to obtain the data. The chi-square test or Fisher's exact test were used to evaluate associations between area and qualitative variables; Student's $t$ test or two proportion comparison test were used to evaluate differences between variables; and a significance level of $5 \%$ adopted. Results: There was significant association between living in Bertioga $(p=0.01)$ and number of pregnancies in the past five years. In São Vicente, 64 (28.3\%) childbearing age women became pregnant whereas in Bertioga there where 109 (38.8\%). There were no statistical significant associations between living in any area and others pregnancy outcomes evaluated. Conclusion: Although no significant association was found, prevalence of low birth weight, preterm delivery and spontaneous abortion and prevalent odds ratio were higher in contaminated area. This study's evidence of a reduced number of pregnancies in contaminated area strengthens the need for additional more in-depth studies in Santos and São Vicente Estuary.

Keywords: Pregnancy outcomes. Environmental pollution. Organochlorine compounds. Heavy metals. Low birth weight. Prematurity.

\section{Introdução}

O desenvolvimento industrial proporcionou ao homem acesso a uma qualidade de vida jamais alcançada que, associado a uma ocupação diferencial do espaço urbano, paradoxalmente aumentou o risco de exposição a substâncias químicas potencialmente deletérias à saúde humana ${ }^{1}$.

Segundo a Companhia de Tecnologia de Saneamento Ambiental - CETESB ${ }^{2}$, no Brasil os sistemas estuarinos de Santos e São Vicente são exemplos de degradação ambiental, ocasionada pela forte pressão sobre estes frágeis ecossistemas, reflexo da intensa ocupação humana que regiões costeiras como elas tendem a apresentar de uma maneira geral.

Na região metropolitana da Baixada Santista, a disponibilidade local de uma desenvolvida infraestrutura de transportes rodoviário, ferroviário e portuário, água e energia elétrica, associada à proximidade com a região metropolitana de São Paulo, levou, a partir da década de 50, à implantação de diversas indústrias de base (siderurgia, petroquímica, fertilizantes) em meio à ampla rede de canais estuarinos e extensos manguezais confinados entre o oceano Atlântico e a Serra do Mar. Esta atividade industrial, de alto potencial poluidor, muitas vezes não acompanhada de medidas de controle, fez dos estuários de Santos e São Vicente grandes receptores de resíduos tóxicos e efluentes contaminados, principalmente com poeiras, metais pesados, organoclorados, bifenilas policloradas (PCBs), hidrocarbonetos policíclicos aromáticos (HPAs), dioxinas e furanos. Os poluentes industriais, juntamente com os resíduos e esgotos do Porto de Santos e das cidades da região, provocam um grave quadro de contaminação ambiental, com significativos reflexos na área social e de saúde pública² ${ }^{2}$.

Os efeitos da exposição crônica a contaminantes industriais difundidos no ambiente, que surgem após um intervalo de tempo variável, constituem riscos para doenças com importantes efeitos populacionais, contrapondo-se às exposições 
aguda e ocupacional, de maior impacto no nível individual ${ }^{3}$.

Há uma preocupação crescente na comunidade científica sobre os efeitos no sistema reprodutivo por exposição das grávidas a produtos químicos. Diversos estudos fornecem evidências de que fetos e bebês provavelmente sejam mais sensíveis às variedades de substâncias ambientais tóxicas do que os adultos ${ }^{4}$.

Existem, na literatura mundial e no Brasil em particular, poucos trabalhos avaliando os riscos à saúde embrio-fetal decorrentes da contaminação ambiental ${ }^{1}$. A identificação de potenciais fatores de risco para a gestante e seu concepto é ação primordial na busca pela redução dos índices de morbidade e mortalidade materna, fetal e neonatal.

Uma recente revisão, feita por Shirangi e colaboradores (2011), trouxe evidências que sugerem associação entre exposição à utilização de pesticidas próximos a residências e aumento de desfechos adversos do sistema reprodutivo. Dentre os desfechos, as malformações congênitas apresentaram uma força de associação maior. Outros desfechos, como baixo peso ao nascer, prematuridade e aborto, embora apresentem associações mais fracas, merecem novas investigações ${ }^{5}$.

Como contribuição para uma aproximação preliminar ao tema, o presente estudo teve por objetivo estimar e comparar a prevalência de eventos relacionados à gravidez (engravidar, baixo peso de nascimento, parto prematuro, aborto espontâneo, natimortalidade, malformações congênitas, e gemelaridade) em bairros de dois municípios (São Vicente e Bertioga) na região do estuário de Santos e São Vicente.

\section{Métodos}

Trata-se de um estudo transversal que fez parte da primeira fase de um projeto mais amplo e extenso intitulado "Estudo Epidemiológico na População Residente na Baixada Santista - Estuário de Santos: Avaliação de Indicadores de Efeito e de Exposição a Contaminantes Ambientais", cujo objetivo foi estimar os efeitos na saúde associados à exposição aos contaminantes ambientais entre os moradores da Baixada Santista - Estuário de Santos e São Vicente.

A identificação das áreas contaminadas utilizadas neste estudo foi feita a partir do relatório da CETESB denominado "Sistema Estuarino de Santos e São Vicente"2, que teve como objetivo avaliar a contaminação da água, dos sedimentos e dos organismos aquáticos do sistema estuarino de Santos e São Vicente e a Baía de Santos, relacionando-a com as fontes potenciais de poluentes na região.

Identificadas as áreas, a amostra das populações exposta e não-exposta foi calculada tendo como base a prevalência de malformações congênitas no Brasil e a população residente por distrito censitário nos bairros, conforme censo de 2000 do Instituto Brasileiro de Geografia e Estatística - IBGE.

Neste estudo preliminar foram selecionados três bairros, em duas áreas distintas (Figura 1). Dois deles, Parque das Bandeiras e Parque das Bandeiras Gleba II, estão localizados em São Vicente (Área 1), na porção continental, em um raio de até $1,5 \mathrm{Km}$ de distância de depósitos de resíduos industriais identificados pela CETESB ${ }^{2}$. Estes depósitos, datados de meados da década de 1960 , contêm metais pesados (principalmente mercúrio e zinco) e organoclorados (como o hexaclorobenzeno $)^{2}$, tendo como rotas de exposições possíveis e completas, segundo critérios da ATSDR, o solo, a água e o $\mathrm{ar}^{6}$. Como região de contraste em relação à contaminação ambiental, mas apresentando características socioeconômicas próximas da região da Baixada Santista, foi escolhido o bairro Jardim Vicente de Carvalho II, localizado em Bertioga (Área 2), ao longo da rodovia Rio-Santos. Foram selecionadas 193 famílias no Parque das Bandeiras, 43 no Parque das Bandeiras Gleba II e 251 no Jardim Vicente de Carvalho II, totalizando 487 domicílios.

Em cada domicílio foi aplicado um questionário estruturado que teve como referência o questionário de morbidade referida desenvolvido por pesquisadores 


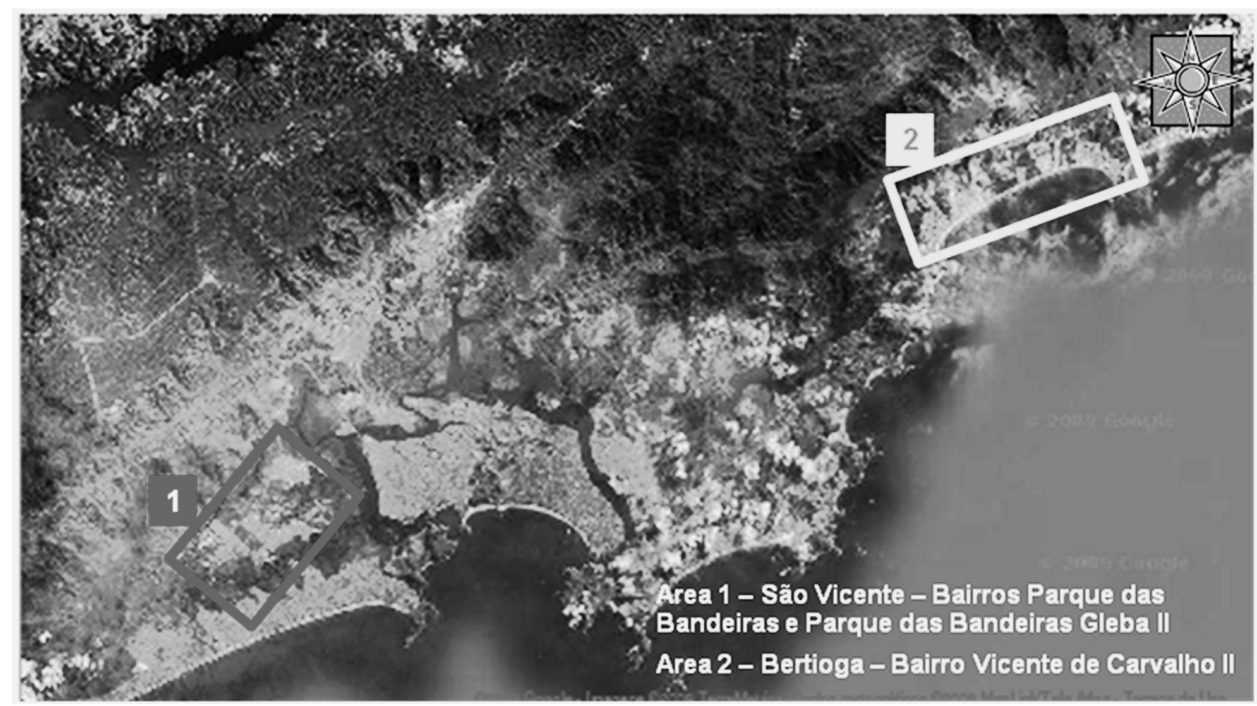

Figura 1 - Áreas avaliadas na região do Estuário de Santos e São Vicente Figure 1 - Areas analyzed in the Estuary region of Santos and São Vicente

do Instituto Nacional do Câncer (INCA) em 2003. Esse questionário foi adaptado às necessidades do projeto de pesquisa $\mathrm{e}$ pré-testado com o objetivo de garantir sua consistência e aplicabilidade. As entrevistas foram realizadas durante os finais de semana (entre março e dezembro de 2007) e, nos casos em que, além do informante, os demais moradores se encontravam no domicílio, os mesmos colaboraram com suas informações pessoais. O estudo foi submetido e aprovado pelo Comitê de Ética Médica em Pesquisa. Após a coleta dos dados foi feita uma análise descritiva das mulheres em idade fértil, entre 15 e 49 anos, conforme classificação do IBGE. Para avaliar os desfechos da gravidez (baixo peso ao nascer - nascido vivo com peso inferior a 2500g; prematuridade - nascidos vivos com idade gestacional inferior a 37 semanas; aborto espontâneo - perda gestacional abaixo de 20 semanas; natimortalidade perda gestacional de 20 semanas ou mais, malformações congênitas e gemelaridade) foram utilizados os dados das mulheres que engravidaram nos últimos cinco anos.

Para testar a associação entre as variáveis qualitativas foi utilizado o teste do qui-quadrado ou teste exato de Fisher. Para comparar proporções foi utilizado o teste de comparação entre duas proporções?
Para testar a diferença entre as variáveis foi utilizado o teste $t$ de Student ou o teste de comparação de duas proporções ${ }^{8}$. Também foram calculadas as prevalências de cada desfecho da gestação por área e a razão de chances prevalente (RCP) entre as áreas.

Foram excluídos das análises os questionários que continham erros de marcação, os que estavam em branco, e quando o entrevistado não sabia informar.

Foi utilizado o SPSS (versão 13.0) e adotado nível de significância igual a 0,05.

\section{Resultados}

Entre as 450 mulheres residentes nos dois bairros de São Vicente e as 508 mulheres no bairro de Bertioga, 226 e 281, respectivamente, estão dentro da faixa etária de mulheres em idade fértil.

A média de idade das mulheres em idade fértil é de 31,70 anos na área de São Vicente, onde $75 \%$ apresentam 40 anos ou menos. $\mathrm{Na}$ área de Bertioga, a média de idade das mulheres em idade fértil é de 29,39 anos, em que $75 \%$ dessas mulheres apresentam 36 anos ou menos. O teste t mostra uma diferença estatisticamente significativa entre as médias das idades da amostra estudada nas duas áreas $(\mathrm{p}=0,02)$.

A Tabela 1 mostra as características 
Tabela 1 - Características sociodemográficas das mulheres em idade fértil segundo as áreas analisadas

Table 1 - Socio-demographic characteristics of women according to areas analyzed

\begin{tabular}{|c|c|c|c|}
\hline & \multicolumn{2}{|c|}{ Áreas - N (\%) } & \multirow{2}{*}{ TOTAL } \\
\hline & São Vicente* & Bertioga* & \\
\hline \multicolumn{4}{|l|}{ Estado Civil } \\
\hline Casada/Mora junto & $141(56,2)$ & $157(53,4)$ & $298(54,7)$ \\
\hline Separada/ Divorciada & $13(5,2)$ & $23(7,8)$ & $36(6,6)$ \\
\hline Solteira & $91(36,3)$ & $111(37,8)$ & $202(37,1)$ \\
\hline Viúva & $6(2,4)$ & $3(1,0)$ & $9(1,7)$ \\
\hline Total & $251(100,0)$ & $294(100,0)$ & $545(100,0)$ \\
\hline \multicolumn{4}{|l|}{ Escolaridade } \\
\hline Analfabeto funcional ${ }^{* *}$ & $5(2,0)$ & $18(6,3)$ & $23(4,3)$ \\
\hline Ensino fundamental & $92(36,9)$ & $152(53,1)$ & $244(45,6)$ \\
\hline Ensino Médio & $131(52,6)$ & $107(37,4)$ & $238(44,5)$ \\
\hline Ensino Superior & $21(8,4)$ & $9(3,1)$ & $30(5,6)$ \\
\hline Total & $249(100,0)$ & $286(100,0)$ & $535(100,0)$ \\
\hline \multicolumn{4}{|l|}{ Trabalho atual } \\
\hline Sim & $115(45,8)$ & $145(49,7)$ & $260(47,9)$ \\
\hline Não & $136(54,2)$ & $147(50,3)$ & $283(52,1)$ \\
\hline Total & $251(100,0)$ & $292(100,0)$ & $543(100,0)$ \\
\hline \multicolumn{4}{|c|}{ Contato com produto químico no trabalho atual } \\
\hline Sim & $12(10,8)$ & $11(7,7)$ & $23(9,1)$ \\
\hline Não & $99(89,2)$ & $132(92,3)$ & $231(90,9)$ \\
\hline Total & $111(100,0)$ & $143(100,0)$ & $254(100,0)$ \\
\hline \multicolumn{4}{|l|}{ Trabalho anterior } \\
\hline Sim & $106(45,1)$ & $151(53,7)$ & $257(49,8)$ \\
\hline Não & $129(54,9)$ & $130(46,3)$ & $259(50,2)$ \\
\hline Total & $235(100,0)$ & $281(100,0)$ & $516(100,0)$ \\
\hline \multicolumn{4}{|c|}{ Contato com produto químico no trabalho anterior } \\
\hline Sim & $7(7,1)$ & $7(5,4)$ & $14(6,1)$ \\
\hline Não & $92(92,9)$ & $122(94,6)$ & $214(93,9)$ \\
\hline Total & $99(100,0)$ & $129(100,0)$ & $228(100,0)$ \\
\hline \multicolumn{4}{|l|}{ Tabagismo atual } \\
\hline Sim & $26(10,4)$ & $58(19,7)$ & $84(15,5)$ \\
\hline Não & $223(89,6)$ & $236(80,3)$ & $459(84,5)$ \\
\hline Total & $249(100,0)$ & $294(100,0)$ & $543(100,0)$ \\
\hline \multicolumn{4}{|l|}{ Tabagismo anterior } \\
\hline Sim & $26(10,5)$ & $38(13,0)$ & $64(11,9)$ \\
\hline Não & $221(89,5)$ & $255(87,0)$ & $476(88,1)$ \\
\hline Total & $247(100,0)$ & $293(100,0)$ & $540(100,0)$ \\
\hline \multicolumn{4}{|l|}{ Consumo de álcool } \\
\hline Sim & $34(13,7)$ & $59(20,1)$ & $93(17,2)$ \\
\hline Não & $214(86,3)$ & $235(79,9)$ & $449(82,8)$ \\
\hline Total & $248(100,0)$ & $294(100,0)$ & $542(100,0)$ \\
\hline \multicolumn{4}{|c|}{ Consumo pregresso de álcool } \\
\hline Sim & $6(2,5)$ & $20(6,9)$ & $26(4,9)$ \\
\hline Não & $238(97,5)$ & $271(93,1)$ & $509(95,1)$ \\
\hline Total & $244(100,0)$ & $291(100,0)$ & $535(100,0)$ \\
\hline
\end{tabular}

* São Vicente = bairros Parque das Bandeiras e Parque das Bandeiras Gleba II; Bertioga = bairro Vicente de Carvalho II.

**Analfabeto funcional: Os declarados analfabetos e aqueles que cursaram até a $2^{\text {a }}$ série do ensino fundamental.

* São Vicente = Parque das Bandeiras and Parque das Bandeiras Gleba II neighborhoods; Bertioga = Vicente de Carvalho II neighborhood.

** Functional Illiteracy: Those who declared themselves illiterate and those with up to 2nd year of elementary school schooling. 
sociodemográficas das mulheres em idade fértil nas áreas analisadas. Em ambas as áreas mais de $50 \%$ dessas mulheres são casadas ou moram com o companheiro. Não há associação significativa entre o local de moradia e o estado civil das mulheres (teste qui-quadrado, $\mathrm{p}=0,37$ ).

Em relação à escolaridade, há uma associação com o local de residência pelo teste do qui-quadrado $(\mathrm{p}=0,00)$. Na área de Bertioga, 53,1\% dos entrevistados têm até o ensino fundamental completo, enquanto na área de São Vicente 36,9\%. Com mais anos de estudo (entre ensino médio e ensino superior), há um maior percentual na área de São Vicente (61\%) em relação à área de Bertioga (40,4\%) (Tabela 1).

Entre as mulheres em idade fértil, 45,8\% e 49,7\% trabalham nas áreas de São Vicente e Bertioga, respectivamente. Não há associação significativa entre as áreas pelo teste exato de Fisher $(p=0,46)$. Das mulheres que trabalham atualmente, poucas têm contato com algum produto químico no trabalho, apenas 10,8\% na área de São Vicente e 7,7\% na área de Bertioga (Tabela 1). Não há associação significativa entre as áreas pelo teste exato de Fisher $(p=0,66)$.

Trabalharam de forma remunerada $45,1 \%$ e $53,7 \%$ das mulheres em idade fértil nas áreas de São Vicente e Bertioga, respectivamente. Não há diferença significativa entre as áreas identificada pelo teste de comparação de duas proporções. Apenas $7,1 \%$ na área de São Vicente e 5,4\% na área de Bertioga das mulheres que trabalhavam tiveram contato com algum produto químico no trabalho (Tabela 1), sem associação significativa entre as áreas segundo o teste exato de Fisher $(p=0,08)$.

Entre as mulheres em idade fértil, $10,4 \%$ fumam na área de São Vicente e $19,7 \%$ na área de Bertioga, apresentando uma diferença significativa pelo teste de comparação de duas proporções $(p=0,01)$. O percentual de mulheres que fumavam em ambas as áreas foi de 10,5\% na área de São Vicente e de 13\% na área de Bertioga (Tabela 1), sem associação significativa com o local de moradia segundo o teste exato de Fisher $(p=0,39)$.
Poucas mulheres relatam consumo de bebida alcoólica nas duas áreas, 13,7\% em São Vicente e 20,1\% em Bertioga, mas há diferença significativa com o local de moradia segundo o teste de comparação de duas proporções $(\mathrm{p}=0,03)$. Do mesmo modo, há associação estatisticamente significativa entre o local de moradia e o consumo passado de bebida alcoólica segundo o teste exato de Fisher $(\mathrm{p}=0,03)$, mas poucas mulheres em idade fértil, 2,5\% na área de São Vicente e 6,9\% na área de Bertioga, relatam ter consumido bebida alcoólica em ambas as áreas (Tabela 1).

$\mathrm{Na}$ área de São Vicente, 64 mulheres $(28,3 \%)$ engravidaram nos últimos cinco anos, enquanto na área de Bertioga foram 109 mulheres (38,8\%) (Tabela 2). Existe diferença estatisticamente significativa entre o número de gestantes e o local de moradia, pelo teste de comparação de duas proporções $(p=0,01)$.

Além de um maior número de mulheres que engravidaram nos últimos cinco anos, a área de Bertioga também apresenta um número maior de gestações quando comparada à de São Vicente. As 64 mulheres que engravidaram na área de São Vicente tiveram 82 gestações nos últimos cinco anos, $\mathrm{e}$ entre as 109 mulheres que engravidaram na área de Bertioga ocorreram 155 gestações no período. A média de gestações por mulher nos últimos cinco anos na área de São Vicente é de 1,28 gestações (desvio padrão de 0,57) e na área de Bertioga de 1,42 (desvio padrão de 0,72 ), não havendo, no entanto, diferença significativa entre as médias pelo teste $\mathrm{t}(\mathrm{p}=0,06)$ (Tabela 2$)$.

A maioria das mulheres disse ter feito acompanhamento pré-natal em suas gestações nos últimos cinco anos: 62 (96,9\%) na área de São Vicente e 103 (95,4\%) na área de Bertioga, sem associação significativa entre o local de moradia e a atenção pré-natal, segundo o teste exato de Fisher $(\mathrm{p}=0,65)$ (Tabela 3).

A Tabela 3 também apresenta os desfechos relacionados à gestação entre as mulheres que engravidaram nos últimos cinco anos nas áreas estudadas. A área de 
Tabela 2 - Distribuição das mulheres que engravidaram e do número de gestações nos últimos cinco anos segundo as áreas analisadas

Table 2 - Number of pregnant women and pregnancies in the past five years according to areas analyzed

\begin{tabular}{lcc}
\hline & \multicolumn{2}{c}{ Áreas - N (\%) } \\
\cline { 2 - 3 } Engravidou & São Vicente* & Bertioga* \\
$\quad$ Sim & $64(28,3)$ & $109(38,8)$ \\
$\quad$ Não & $162(71,7)$ & $172(61,2)$ \\
$\quad$ Total & $226(100,0)$ & $281(100,0)$ \\
Média de gestações & 1,28 & 1,42 \\
Desvio Padrão & 0,57 & 0,72 \\
Mínimo & 1 & 1 \\
Máximo & 4 & 5 \\
Percentil 25 & 1,00 & 1,00 \\
Percentil 50 & 1,00 & 1,00 \\
Percentil 75 & 1,00 & 2,00 \\
\hline
\end{tabular}

* São Vicente: bairros Parque das Bandeiras e Parque das Bandeiras Gleba II; Bertioga: bairro Vicente de Carvalho II.

* São Vicente: Parque das Bandeiras and Parque das Bandeiras Gleba II neighborhoods; Bertioga: Vicente de Carvalho II neighborhood.

São Vicente tem um percentual maior de mulheres que sofreram aborto espontâneo do que a área de Bertioga, não sendo significativamente diferente entre as áreas segundo o teste de comparação de duas proporções $(\mathrm{p}=0,24)$. Pelo cálculo da RCP, a chance de ocorrência de abortos na área de São Vicente é 1,58 vez maior do que na área de Bertioga.

Na área de SãoVicente há um percentual maior de bebês prematuros $(8,1 \%)$ do que na área de Bertioga (5,7\%). A RCP mostra 1,42 vez mais chance de ocorrência de nascimentos prematuros na área de São Vicente do que na área de Bertioga, mas a diferença não é significativa segundo o teste de comparação de duas proporções ( $\mathrm{p}=0,78$ ). Na área de São Vicente, 4 mulheres $(6,9 \%)$ tiveram filho(s) com peso inferior a $2.500 \mathrm{~g}$, enquanto na área de Bertioga foram 5 mulheres $(4,7 \%)$ (Tabela 3$)$. A RCP mostra 1,47 vez mais chance de ocorrência de nascimento com baixo peso na área de São Vicente do que na área de Bertioga, mas também não há diferença significativa segundo o teste de comparação de duas proporções ( $\mathrm{p}=0,82)$.

Em relação à freqüência de natimortos, só houve um caso nos últimos cinco anos entre os domicílios entrevistados, ocorrido na área de São Vicente. Também só houve um caso de nascimento gemelar nos últimos cinco anos ocorrido na área de Bertioga. Houve poucos casos em que bebês apresentaram malformações congênitas, um caso na área de São Vicente $(1,6 \%)$ e três casos na área de Bertioga $(2,8 \%)$.

\section{Discussão}

A área de Bertioga apresentou um percentual maior de mulheres em idade fértil que ficaram grávidas nos últimos cinco anos em relação à área de São Vicente, com diferença significativa entre as áreas. Em relação à média de gestações por mulher que ficou grávida nos últimos cinco anos, embora maior na área de Bertioga do que na de São Vicente, não foram encontradas diferenças estatisticamente significantes. Existe no Brasil uma tendência de declínio da fecundidade, verificada nos últimos censos demográficos do IBGE. No país como um todo, a transição da fecundidade está próxima ao nível de reposição populacional. Contudo, existem diferenças internas bastante signi- 
Tabela 3 - Acompanhamento pré-natal e desfechos da gravidez entre as mulheres que ficaram grávidas nos últimos cinco anos segundo as áreas analisadas

Table 3 - Prenatal care and pregnancy outcomes among women who became pregnant in the past five years according to areas analyzed

\begin{tabular}{|c|c|c|c|}
\hline & \multicolumn{2}{|c|}{ Áreas (N (\%) } & \multirow{2}{*}{ TOTAL } \\
\hline & São Vicente* & Bertioga* & \\
\hline \multicolumn{4}{|c|}{ Acompanhamento pré-natal } \\
\hline Sim & $62(96,9)$ & $103(95,4)$ & $165(95,9)$ \\
\hline Não & $2(3,1)$ & $5(4,6)$ & $7(4,1)$ \\
\hline Total & $64(100,0)$ & $108(100,0)$ & $172(100,0)$ \\
\hline \multicolumn{4}{|c|}{ Aborto espontâneo } \\
\hline Sim & $14(23,3)$ & $15(14,7)$ & $29(17,9)$ \\
\hline Não & $46(76,7)$ & $87(85,3)$ & $133(82,1)$ \\
\hline Total & $60(100,0)$ & $102(100,0)$ & $162(100,0)$ \\
\hline \multicolumn{4}{|l|}{ Prematuridade } \\
\hline Prematuro & $5(8,1)$ & $6(5,7)$ & $11(6,6)$ \\
\hline Termo & $57(91,9)$ & $99(94,3)$ & $156(93,4)$ \\
\hline Total & $62(100,0)$ & $105(100,0)$ & $167(100,0)$ \\
\hline \multicolumn{4}{|c|}{ Baixo peso ao nascer } \\
\hline$<2.500 \mathrm{~g}$ & $4(6,9)$ & $5(4,7)$ & $9(5,5)$ \\
\hline$\geq 2.500 \mathrm{~g}$ & $54(93,1)$ & $101(95,3)$ & $155(94,5)$ \\
\hline Total & $58(100,0)$ & $106(100,0)$ & $164(100,0)$ \\
\hline
\end{tabular}

* São Vicente: bairros Parque das Bandeiras e Parque das Bandeiras Gleba Il; Bertioga: bairro Vicente de Carvalho II.

* São Vicente: Parque das Bandeiras and Parque das Bandeiras Gleba II neighborhoods; Bertioga: Vicente de Carvalho II neighborhood.

ficativas nas taxas de fecundidade, por estas estarem correlacionadas negativamente com fatores socioeconômicos, como renda e escolaridade ${ }^{9}$, fatores estes que, no presente estudo, apresentaram um nível pior na área de Bertioga do que na de São Vicente.

Os sistemas reprodutivo feminino e masculino são susceptíveis a fatores ambientais, tendo impacto no desenvolvimento dos tecidos e também nas funções reprodutivas dos adultos, como a diminuição da fecundidade de homens e mulheres ${ }^{10,11}$. A evidência entre exposição a poluentes ambientais e fertilidade humana é controversa e os resultados pouco consistentes ${ }^{12}$, porém, estudos experimentais têm demonstrado esta associação ${ }^{13}$. Guillette e Edwards (2008) relatam que contaminantes ambientais, incluindo pesticidas, agentes farmacêuticos e poluentes industriais, desempenham papéis de disruptores endócri- nos, alterando o desempenho reprodutivo de populações selvagens de peixes, anfíbios, répteis e aves ${ }^{13}$.

A associação entre contaminantes ambientais e desfechos na gestação, tais como aborto espontâneo, parto prematuro e baixo peso de nascimento, tem sido investigada por diversos autores. Um estudo de revisão feito por Windham e Fenster ${ }^{14}$ mostrou associação com incremento de risco para poluição atmosférica, metais pesados e pesticidas, entre outros. Para pesticidas organoclorados, os autores relataram uma relação dose-resposta entre aumento dos níveis de dicloroetileno (DDE) e prematuridade.

O conhecimento sobre os efeitos de organoclorados na saúde humana é limitado e os resultados são normalmente conflitantes ${ }^{10}$. Em uma extensa revisão de estudos epidemiológicos, os autores relataram que populações expostas a altas 
concentrações de PCBs ou DDE apresentam anormalidades reprodutivas, incluindo abortos espontâneos e redução do peso de nascimento. Também relataram que em outros estudos, com exposição às médias e baixas concentrações de PCBs ou DDE, não foram encontradas associações com estes mesmos desfechos adversos da gestação.

Outra revisão analisou estudos entre exposição ao Dicloro-Difenil-Tricloroetano (DDT) e/ou DDE e desfechos adversos da gestação e também encontrou resultados conflitantes ${ }^{14}$. Os autores relataram que alguns estudos mostraram associações entre estes pesticidas organoclorados com o aumento da ocorrência de partos prematuros e diminuição do peso ao nascer, e também outros que não mostraram associações com estes mesmos desfechos da gestação.

Abortos, nascimentos prematuros e com baixo peso apresentaram prevalência e razões de chances maiores na área de São Vicente do que na de Bertioga, embora sem diferenças significativas entre as áreas.

Estes resultados podem ser valorizados, já que na área contaminada não há associação com outros fatores que possam interferir na gestação, tais como atenção pré-natal, exposição ocupacional, consumo de bebida alcoólica e tabagismo.

O objetivo principal da atenção pré-natal é acolher a mulher desde o início da gestação, assegurando o nascimento de uma criança saudável e o bem-estar materno e neonatal, com ações que integrem todos os níveis de atenção à saúde ${ }^{15}$. A má assistência pré-natal é um importante fator de risco para mortalidade perinatal ${ }^{16}$, parto prematuro e baixo peso de nascimento ${ }^{17}$. Laurenti e Buchalla ${ }^{18}$ demonstraram que mães que tiveram sete ou mais consultas de pré-natal apresentaram uma menor mortalidade perinatal. Dados da Fundação SEADE mostram uma grande diferença percentual entre os municípios de São Vicente $(70,86 \%)$ e Bertioga (34,05\%) em relação ao número de mães que tiveram sete ou mais consultas de pré-natal. Quase todas as mulheres que engravidaram nos últimos cinco anos, em ambas as áreas, fizeram acompanhamento pré-natal, mas o questionário utilizado neste estudo não previa avaliar a quantidade de consultas, o local onde foram realizados os atendimentos e nem a qualidade destes atendimentos.

Em contraposição à contaminação ambiental não-ocupacional em gestantes, a exposição ocupacional é um importante fator que deve ser controlado ${ }^{1}$. A exposição ocupacional a produtos químicos tem sido relatada como um importante fator de risco na gestação, interferindo no perfil de morbimortalidade perinatal ${ }^{12}$. No presente estudo poucas mulheres estiveram expostas aos produtos químicos no local de trabalho em ambas as áreas investigadas, não sendo, portanto, um fator relevante na associação entre os desfechos e as áreas analisadas.

A área de Bertioga apresentou um maior percentual de mulheres em idade fértil com exposição atual e pregressa a bebidas alcoólicas, havendo diferença significativa em relação à área de São Vicente, embora não tenha sido possível investigar se o consumo ocorreu durante o período gestacional. $\mathrm{O}$ álcool é uma substância com importante efeito teratogênico, que interfere no desenvolvimento fetal. Freire e colaboradores ${ }^{19}$ observaram que o uso de álcool na gravidez esteve associado à restrição do crescimento fetal, sendo os do sexo feminino aparentemente mais susceptível aos seus efeitos. Os autores relatam que $10 \%$ das crianças nascidas com restrição de crescimento intra-uterino nos EUA tiveram exposição fetal ao álcool e, ainda, que $11 \%$ dos pacientes com deficiência mental sofreram os efeitos teratógenos do álcool.

A gestante tabagista provoca prejuízos à sua própria saúde, como também à saúde fetal. Os efeitos do cigarro na gestação incluem: baixo peso de nascimento, partos prematuros e abortos espontâneos ${ }^{20}$. A exposição passiva ao tabaco também deve ser considerada, já que estudos têm concluído pequeno efeito, porém consistente, na redução do peso de nascimento e na ocorrência de partos prematuros ${ }^{14}$. A área de Bertioga apresentou um maior percentual de mulheres em idade fértil com exposição atual 
e pregressa ao fumo, embora não tenha sido possível estabelecer se o consumo ocorreu durante o período gestacional.

Estudos mostram associação entre exposição a contaminantes ambientais e aborto espontâneo ${ }^{21}$, natimortalidade ${ }^{22}$, malformações congênitas ${ }^{23}$ e gemelaridade $^{24}$;contudo, no presente trabalho não foi possível avaliar estes desfechos devido à ausência de registros no período estudado.

Em nenhum dos desfechos relacionados à gravidez houve perda de informação superior a $20 \%$. As perdas de informação, discriminadas por variável, foram consideradas pouco expressivas: na análise do acompanhamento pré-natal por mulher houve perda de uma informação $(0,58 \%)$; no número de gestações por mulher, nove $(5,20 \%)$; na gemelaridade, quatro $(2,31 \%)$; mulheres com aborto espontâneo, 11 informações (6,36\%); recém-nascidos com baixo peso e natimortos, nove informações cada $(5,20 \%)$; bebês prematuros, seis $(3,47 \%)$; e no número de bebês com malformações, cinco informações $(2,89 \%)$.

Estudos retrospectivos são mais susceptíveis ao viés de memória ${ }^{25}$, embora no presente estudo os desfechos relativos à gestação investigados tiveram um período de curta duração, menor que cinco anos. Além disso, em mais de 63\% dos domicílios entrevistados as próprias mulheres que estiveram grávidas foram responsáveis pelas informações, o que também reduz significativamente o viés de memória do entrevistado.
Os bairros avaliados em São Vicente estão próximos a depósitos clandestinos de resíduos industriais, contaminados principalmente com hexaclorobenzeno - $\mathrm{HCB}$, hexaclorobutadieno - HCBD, tetraclorobenzeno, pentaclorobenzeno, clorofórmio, percloroetileno e tetracloreto de carbono ${ }^{2}$.

Entretanto, a presença de contaminantes no ambiente não é suficiente para caracterizar a contaminação ou exposição de determinada população. O contato é fundamental para que se defina a presença de uma rota de contaminação. Além do contato, a concentração da substância nos compartimentos ambientais e a duração do contato com estas substâncias são fundamentais para determinar o risco de manifestações clínicas adversas nos indivíduos expostos.

\section{Conclusões}

As prevalências e as razões de chance prevalentes de baixo peso ao nascer, nascimentos prematuros e abortos espontâneos foram maiores nas áreas contaminadas, embora sem associações significativas. Embora o desenho do estudo não permita inferências causais, há evidências suficientes da diminuição do número de gestações nas áreas contaminadas, reforçando a necessidade de aprofundamento de estudos na região do estuário de Santos e São Vicente.

\section{Referências}

1. Leite JCL, Schüller-Faccini L. Defeitos congênitos em uma região de mineração de carvão. Rev Saúde Pública. 2001; 35(2): 136-41.

2. CETESB. Sistema Estuarino de Santos e São Vicente. Relatório Técnico. São Paulo; 2001. 141p.

3. Castilla EE, Campaña H, Camelo JS. Economic activity and congenital anomalies: an ecologic study in Argentina. ECLAMC ECOTERAT Group. Environ Health Perspect 2000; 108(3): 193-7.
4. Thakur JS, Prinja S, Singh D, Rajwanshi A, Prasad R, Parwana HK, et al. Adverse reproductive and child health outcomes among people living near highly toxic waste water drains in Pujab, India. J Epidemiol Community Health 2010; 64: 148-54.

5. Shirangi A, Nieuwenhuijsen M, Vienneau D, Holman CDJ. Living near agricultural pesticide applications and the risk of adverse reproductive outcomes: a review of the literature. Paedriatic and Perinatal Epidemiology 2011; 25: 172-91. 
6. ATSDL - Agency for Toxic Substances \& Disease Registry. Public Health Assessment Guidance Manual [Internet]. Atlanta: Public Health Assessment Guidance Manual; 2005. Disponível em http://www.atsdr.cdc.gov/hac/ PHAManual/toc.html. [Acessado em 20 de julho de 2011]

7. Spiegel S. Estatística não paramétrica. São Paulo: Editora McGraw-Hill do Brasil; 1981.

8. Calleri-Jacques SM. Bioestatística: Princípios e Aplicações. Porto Alegre: Ed. ARTMED; 2003.

9. Berquó E, Cavenaghi S. Fertility in decline: a brief note on the decrease in the number of births in Brazil. Novos Estud-CEBRAP 2006; 74: 11-5.

10. Toft G, Hagmar L, Giwercman A, Bonde JP. Epidemiological evidence on reproductive effects of persistent organochlorines in humans. Reproductive Toxicology 2004; 19: 5-26.

11. Woodruff TK, Walker CL. Fetal and early postnatal environmental exposures and reproductive health effects in the female. Fertility and Sterility 2008; 89(S1): 47-51.

12. Younglai EV, Holloway AC, Foster WG. Environmental and occupational factors affecting fertility and IVF success. Hum Reprod Update 2005; 11(1): e43-57.

13. Guillette Jr LJ, Edwards TM. Environmental influences on fertility: can we learn lessons from studies of wildlife? Fertility and Sterility 2008; 89(S1): e21-4.

14. Windham G, Fenster L. Environmental contaminants and pregnancy outcomes. Fertility and Sterility 2008; 89(S1): e111-6.

15. Brasil. Ministério da Saúde. Secretaria de Atenção à Saúde. Departamento de Ações Programáticas Estratégicas. Área Técnica de Saúde da Mulher. Prénatal e Puerpério: atenção qualificada e humanizada - manual técnico. Série A. Normas e Manuais Técnicos. Brasília; 2005. 163p.
16. De Lorenzi DRS, Tanaka ACA, Bozzetti MC, Ribas FE, Weissheimer L. A natimortalidade como indicador de saúde perinatal. Cad Saúde Pública 2001; 17(1): 141-6.

17. Belford P. Medicina Preventiva: Assistência Pré-natal. In: Rezende J (Org.). Obstetrícia. 10. ed. Rio de Janeiro: Guanabara-Koogan; 2005. p. 268-86.

18. Laurenti R, Buchalla CM. Estudo da morbidade e da mortalidade perinatal em maternidades: II Mortalidade perinatal segundo peso ao nascer, idade materna, assistência pré-natal e hábito de fumar da mãe. Rev Saúde Pública 1985; 19: 225-32.

19. Freire TM, Machado JC, Melo EV, Melo DG. Efeitos do consumo de bebida alcoólica sobre o feto. Rev Bras Ginecol Obstet 2005; 27(7): 376-81.

20. Leopércio W, Gigliotti A. Tabagismo e suas peculiaridades durante a gestação: uma revisão crítica. $J$ Bras Pneumol 2004; 30(2): 176-185.

21. Smrcka V, Leznarova D. Environmental pollution and the occurrence of congenital defects in a 15-year period in a south Moravian district. Acta Chir Plast 1998; 40(4): 1124.

22. Pereira LAA, Loomis D, Conceição GMS, Braga ALF, Arcas RM, Kishi HS, et al. Association between air pollution and intrauterine mortality in São Paulo, Brazil. Environ Health Perspect 1998; 106(6): 325-9.

23. Gilboa SM, Mendola P, Olshan AF, Langlois PH, Savitz DA, Loomis D et al. Relation between ambient air quality and selected birth defects, seven county study, Texas, 1997-2000. Am J Epidemiol 2005; 162(3): 238-52.

24. Obi-Osius N, Misselwitz B, Karmaus W, Witten J. Twin frequency and industrial pollution in different regions of Hesse, Germany. Occup Environ Med 2004; 61(6): 482-7.

25. Schoeps D, Almeida MF, Alencar GP, França Jr I, Novaes HMD, Siqueira AAF et al. Fatores de risco para mortalidade neonatal precoce. Rev Saúde Pública 2007; 41(6): 1013-22. 